\title{
Quantum Cost Optimization for Reversible Sequential Circuit
}

\author{
Md. Selim Al Mamun \\ Jatiya Kabi Kazi Nazrul Islam University \\ Trishal, Mymensingh-2220, Bangladesh
}

\author{
David Menville \\ Pascack Valley High School \\ New Jersey, United States of America
}

\begin{abstract}
Reversible sequential circuits are going to be the significant memory blocks for the forthcoming computing devices for their ultra low power consumption. Therefore design of various types of latches has been considered a major objective for the researchers quite a long time. In this paper we proposed efficient design of reversible sequential circuits that are optimized in terms of quantum cost, delay and garbage outputs. For this we proposed a new $3 * 3$ reversible gate called SAM gate and we then design efficient sequential circuits using SAM gate along with some of the basic reversible logic gates.
\end{abstract}

\section{Keywords-Flip-flop; Garbage Output; Reversible Logic; Quantum Cost}

\section{INTRODUCTION}

In recent years, reversible computing has emerged as a promising technology. The primary reason for this is the increasing demands for lower power devices. In the early 1960s R. Landauer [1] demonstrated that losing bits of information causes loss of energy. Information is lost when an input cannot be recovered from its output. In 1973 C. H. Bennett [2] showed that energy dissipation problem can be avoided if the circuits are built using reversible logic gates.

Reversible logic has the feature to generate one to one correspondence between its input and output. As a result no information is lost and there is no loss of energy [3]. Although many researchers are working in this field, little work has been done in the area of sequential reversible logic. In the current literature on the design of reversible sequential circuits, the number of reversible gates is used as a major metric of optimization [4]. The number of reversible gates is not a good metric of optimization as reversible gates are of different type and have different quantum costs [5]. In this paper, we presented new designs of reversible sequential circuits that are efficient in terms of quantum cost, delay and the number of garbage outputs.

This paper is organized as follows: Section 2 presents some basic definitions related to reversible logic. Section 3 describes some basic reversible logic gates and their quantum implementation. Section 4 introduces our proposed gate 'Selim Al Mamun' (SAM) gate. Section 5 describes the logic synthesis of sequential circuits and comparisons with other researchers. Finally this paper is concluded with the Section 6.

\section{BASIC DEFINITIONS}

In this section, some basic definitions related to reversible logic are presented. We formally define reversible gate, garbage output, delay in reversible circuit and quantum cost of reversible in reversible circuit.

\section{A. Reversible Gate}

A Reversible Gate is a k-input, k-output (denoted by $\mathrm{k}^{*} \mathrm{k}$ ) circuit that produces a unique output pattern for each possible input pattern [6]. If the input vector is $I v$ where $I v=\left(I_{1, j}, I_{2, j}\right.$, $\left.I_{3, j}, \ldots, I_{k-1, j}, I_{k, j}\right)$ and the output vector is $O v$ where $O v=$ $\left(O_{1, j}, O_{2, j}, O_{3, j}, \ldots, O_{k-1, j}, O_{k, j}\right)$, then according to the definition, for each particular vector $j, I v \leftrightarrow O v$.

\section{B. Garbage Output}

Every gate output that is not used as input to other gates or as a primary output is garbage. Unwanted or unused outputs which are needed to maintain reversibility of a reversible gate (or circuit) are known as Garbage Outputs. The garbage output of Feynman gate [7] is shown in Fig. 1 with *.

\section{Delay}

The delay of a logic circuit is the maximum number of gates in a path from any input line to any output line. The definition is based on two assumptions: (i) Each gate performs computation in one unit time and (ii) all inputs to the circuit are available before the computation begins.

In this paper, we used the logical depth as measure of the delay proposed by Mohammadi and Eshghi [8]. The delay of each $1 \times 1$ gate and $2 \times 2$ reversible gate is taken as unit delay 1 . Any $3 \times 3$ reversible gate can be designed from $1 \times 1$ reversible gates and $2 \times 2$ reversible gates, such as CNOT gate, Controlled$\mathrm{V}$ and Controlled- $\mathrm{V}^{+}$gates ( $\mathrm{V}$ is a square-root-of NOT gate and $\mathrm{V}^{+}$is its hermitian). Thus, the delay of a $3 \times 3$ reversible gate can be computed by calculating its logical depth when it is designed from smaller $1 \times 1$ and $2 \times 2$ reversible gates.

\section{Quantum Cost}

The quantum cost of a reversible gate is the number of $1 \times 1$ and $2 \times 2$ reversible gates or quantum gates required in its design. The quantum costs of all reversible $1 \times 1$ and $2 \times 2$ gates are taken as unity [9]. Since every reversible gate is a combination of $1 \times 1$ or $2 \times 2$ quantum gate, therefore the quantum cost of a reversible gate can be calculated by counting the numbers of NOT, Controlled-V, Controlled- $\mathrm{V}^{+}$and CNOT gates used.

\section{QUANTUM ANALYSIS OF DIFFERENT REVERSIBLE GATES}

Every reversible gate can be calculated in terms of quantum cost and hence the reversible circuits can be measured in terms 
of quantum cost. Reducing the quantum cost from reversible circuit is always a challenging one and works are still going on in this area. This section describes some popular reversible gates and quantum equivalent diagram of each reversible gate.

\section{A. Feynman Gate}

Let $I_{v}$ and $O_{v}$ are input and output vector of a $2 * 2$ Feynman gate where $I_{v}$ and $O_{v}$ are defined as follows: $I_{v}=(A, B)$ and $O_{v}$ $=(P=A, Q=A \oplus B)$. The quantum cost of Feynman gate is 1. The block diagram and equivalent quantum representation for a $2 * 2$ Feynman gate are shown in Fig. 1.

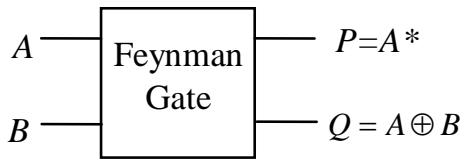

(a)

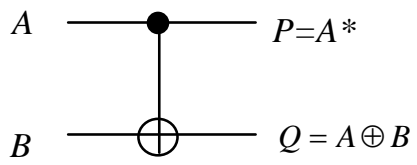

(b)

Fig. 1. (a) Block diagram of $2 \times 2$ Feynman gate and (b) Equivalent quantum representation

\section{B. Double Feynman Gate}

Let $I_{v}$ and $O_{v}$ are input and output vector of a $3 * 3$ Double Feynman gate (DFG) where $I_{v}$ and $O_{v}$ are defined as follows: $I_{v}$ $=(A, B, C)$ and $O_{v}=(P=A, Q=A \oplus B, R=A \oplus C)$. The quantum cost of Double Feynman gate is 2 [10]. The block diagram and equivalent quantum representation for $3 * 3$ Double Feynman gate are shown in Fig. 2.

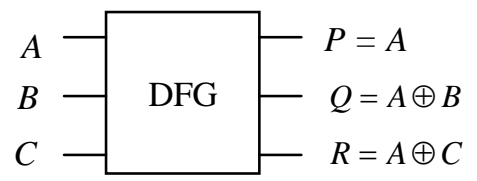

(a)

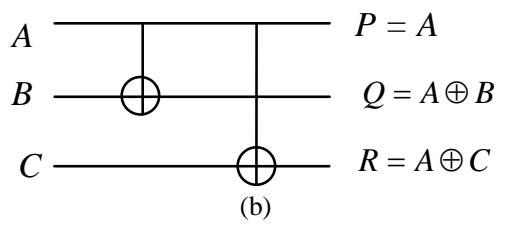

Fig. 2. (a) Block diagram of $3 \times 3$ Double Feynman gate and (b) Equivalent quantum representation.

\section{Toffoli Gate}

The input vector, $I_{v}$ and output vector, $O_{v}$ for $3 * 3$ Toffoli gate (TG) [11] can be defined as follows: $I_{v}=(A, B, C)$ and $O_{v}$ $=(P=A, Q=B, R=A B \oplus C)$. The quantum cost of Toffoli gate is 5 .

The block diagram and equivalent quantum representation for $3 * 3$ Toffoli gate are shown in Fig. 3 .

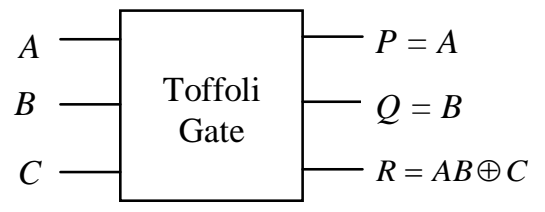

(a)

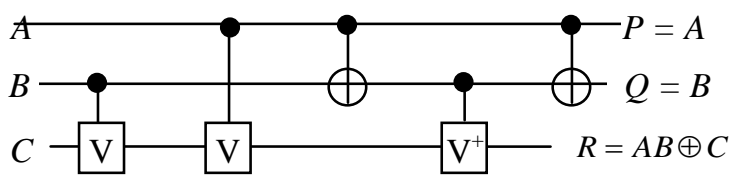

(b)

Fig. 3. (a) Block diagram of $3 * 3$ Toffoli gate and (b) Equivalent quantum representation.

\section{Frekdin Gate}

The input vector, $I_{v}$ and output vector, $O_{v}$ for $3 * 3$ Fredkin gate (FRG) [12] can be defined as follows: $I_{v}=(A, B, C)$ and $\mathrm{O}_{\mathrm{v}}=(P=A, Q=\bar{A} B \oplus A C, R=\bar{A} C \oplus A B)$. The quantum cost of Frekdin gate is 5 . The block diagram and equivalent quantum representation for $3 * 3$ Fredkin gate are shown in Fig. 4.

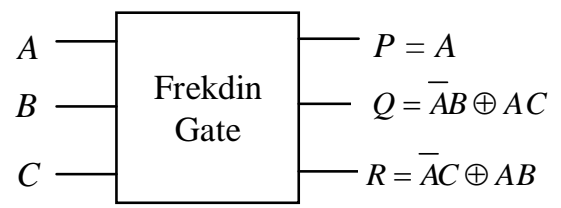

(a)

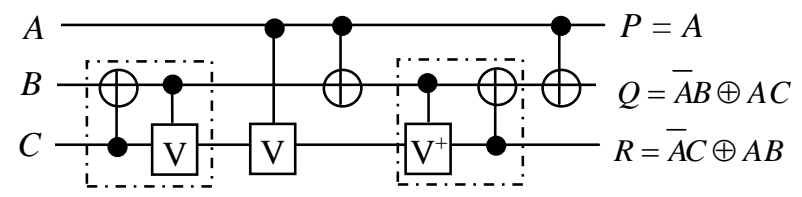

(b)

Fig. 4. (a) Block diagram of $3 * 3$ Frekdin gate and (b) Equivalent quantum representation

\section{E. Peres Gate}

The input vector, $I_{v}$ and output vector, $O_{v}$ for $3 * 3$ Peres gate (PG)[13] can be defined as follows: $I_{v}=(A, B, C)$ and $O_{v}=(P$ $=A, Q=A \oplus B, R=A B \oplus C)$. The quantum cost of Peres gate is 4 . The block diagram and equivalent quantum representation for $3 * 3$ Peres gate are shown in Fig. 5.

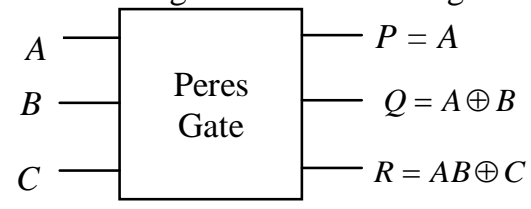

(a)

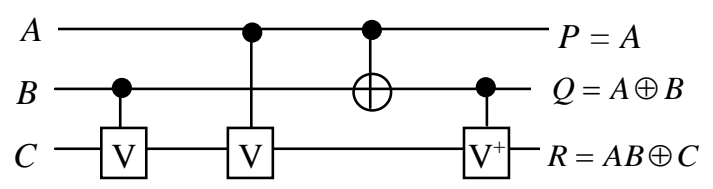

(b)

Fig. 5. Block diagram of $3 * 3$ Peres and (b) Equivalent quantum representation 


\section{PROPOSED SAM GATE}

After The input vector, $I_{v}$ and output vector, $O_{v}$ for $3 * 3$ SAM Gate is defined as follows: $I_{v}=(A, B, C)$ and $O_{\mathrm{v}}=($ $P=\bar{A}, Q=\bar{A} B \oplus A \bar{C}, R=\bar{A} C \oplus A B)$. The block diagram of a $3 * 3$ SAM gate is shown in Fig. 6.

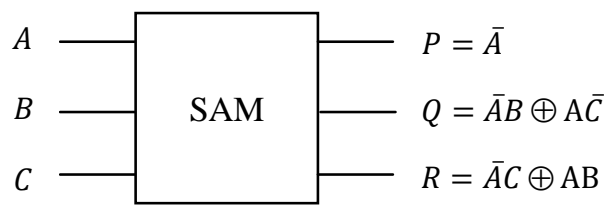

Fig. 6. Block diagram of a $3 * 3 \mathrm{SAM}$ gate

The truth table for a 3x3 SAM gate is shown in Table I.

TABLE I. TRUTH TABLE FOR $3 * 3$ SAM GATE

\begin{tabular}{|c|c|c|c|c|c|}
\hline $\mathbf{A}$ & $\mathbf{B}$ & $\mathbf{C}$ & $P=\bar{A}$ & $Q=\bar{A} B \oplus \mathrm{A} \bar{C}$ & $R=\bar{A} C \oplus \mathrm{AB}$ \\
\hline 0 & 0 & 0 & 1 & 0 & 0 \\
\hline 0 & 0 & 1 & 1 & 0 & 1 \\
\hline 0 & 1 & 0 & 1 & 1 & 0 \\
\hline 0 & 1 & 1 & 1 & 1 & 1 \\
\hline 1 & 0 & 0 & 0 & 1 & 0 \\
\hline 1 & 0 & 1 & 0 & 0 & 0 \\
\hline 1 & 1 & 0 & 0 & 1 & 1 \\
\hline 1 & 1 & 1 & 0 & 0 & \\
\hline
\end{tabular}

We can verify from the corresponding truth table of the SAM gate that the output and input vectors have one to one mapping between them which satisfies the condition of reversibility of a gate. We can see from Table $I$ that the 8 different input and output vectors unique means they have one to one mapping them. So the proposed gate satisfies the condition of reversibility.

The Equivalent quantum representation of the SAM gate and minimization of quantum cost are shown in Fig 7(a) through $7(\mathrm{~d})$. The quantum cost of SAM gate is 4 .

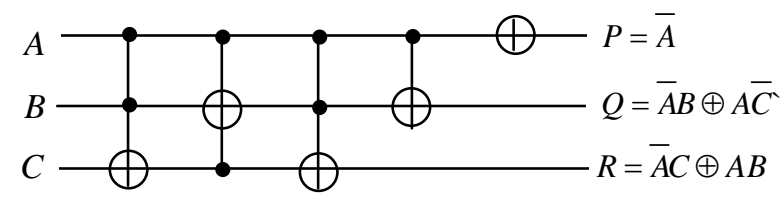

(a)

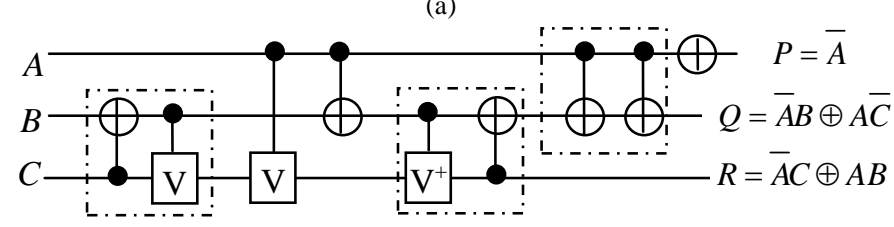

(b)

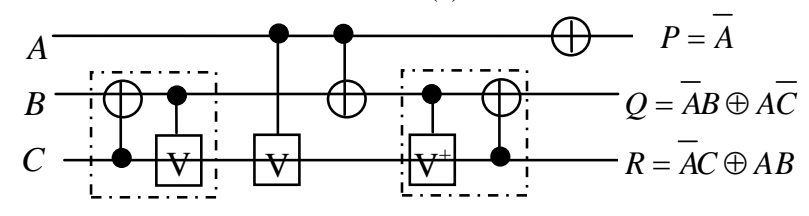

(c)

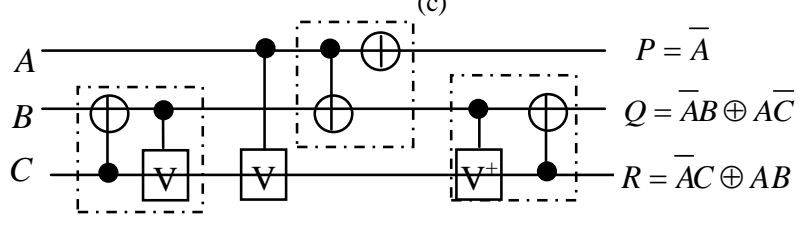

(d)

Fig. 7. Quantum cost of proposed SAM gate.

If we give 0 to $3^{\text {rd }}$ input then we get NOT of $1^{\text {st }}$ input in $1^{\text {st }}$ output, OR or $1^{\text {st }}$ and $2^{\text {nd }}$ inputs in $2^{\text {nd }}$ output and AND of $1^{\text {st }}$ and $2^{\text {nd }}$ inputs in $3^{\text {rd }}$ output. This operation is shown in Fig. 8. So this gate can be used as two input universal gate.

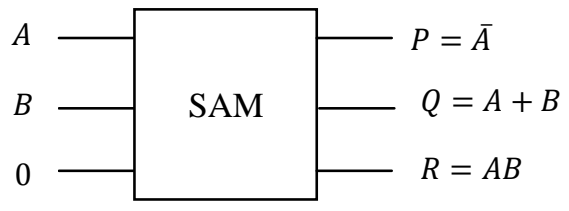

Fig. 8. SAM gate as two input universal gate.

\section{DESIGN AND Synthesis OF REVERSIBLE SEQUENTIAL CIRCUITS}

In this section, we presented novel designs of reversible flip-flops that are optimized in terms of quantum cost, delay and garbage outputs.

\section{A. The SR Flip-Flop}

For SR flip-flop we modified the Peres gate. The modified Peres gate is shown in figure 9 .

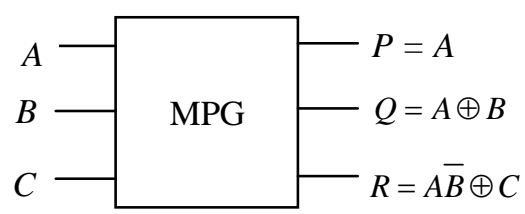

(a)

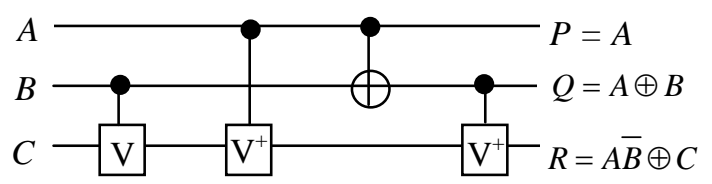

(b)

Fig. 9. (a) Block diagram of $3 * 3$ MPG and (b) Equivalent quantum represenation 
The characteristic equation of SR flip-flop is $Q=S+\bar{R} Q$. The SR flip-flop can be realized by a modified Peres gate (MPG). It can be mapped with the MPG by giving Q, R and S respectively in $1^{\text {st }}, 2^{\text {nd }}$ and $3^{\text {rd }}$ inputs of the MPG. Fig. 10 shows the proposed design of SR flip-flop with $Q$ and $\bar{Q}$ outputs.

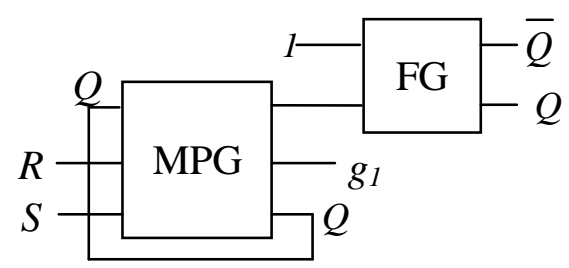

Fig. 10. Proposed design of SR flip-flop with $Q$ and $\bar{Q}$ outputs

The proposed SR flip-flop with $Q$ and $\bar{Q}$ outputs has quantum cost 5 , delay 5 and has the bare minimum of 1 garbage bit. The proposed design of SR flip-flop achieves improvement ratios of $50 \%$ in terms of quantum cost, delay and garbage outputs compared to the design presented by Rice 2008 [14]. The improvement ratios compared to the design presented in Thapliyal et al.2010 [15] are 37\%, 37\% and 50\% in terms quantum cost, delay and garbage outputs. The comparisons of our SR flip-flop (with $Q$ and $\bar{Q}$ outputs) design with existing designs in literature are summarized in Table II.

TABLE II. COMPARISONS OF DIFFERENT TYPES OF SR FLIP-FLOPS WITH Q AND $\bar{Q}$ OUTPUTS

\begin{tabular}{|l|c|c|c|}
\hline \multirow{2}{*}{ SR flip-flop design } & \multicolumn{3}{|c|}{ Cost Comparison } \\
\cline { 2 - 4 } & $\begin{array}{c}\text { Quantum } \\
\text { Cost }\end{array}$ & Delay & $\begin{array}{c}\text { Garbage } \\
\text { Outputs }\end{array}$ \\
\hline Proposed & 5 & 5 & 1 \\
\hline Existing [14] & 10 & 10 & 2 \\
\hline Existing [15] & 8 & 8 & 2 \\
\hline Improvement(\%) w.r.t. [14] & 50 & 50 & 50 \\
\hline Improvement(\%) w.r.t. [15] & 37 & 37 & 50 \\
\hline
\end{tabular}

This SR flip-flop design does not have enable signal (clock) and hence is not gated in nature. We proposed a design of gated SR flip-flop that can be realized by one MPG gate, one SAM and one FG gate. Another FG is needed to copy and produce the complement of Q. So we used a DFG instead of two FGs. The proposed gated SR flip-flop is shown in Fig. 11.

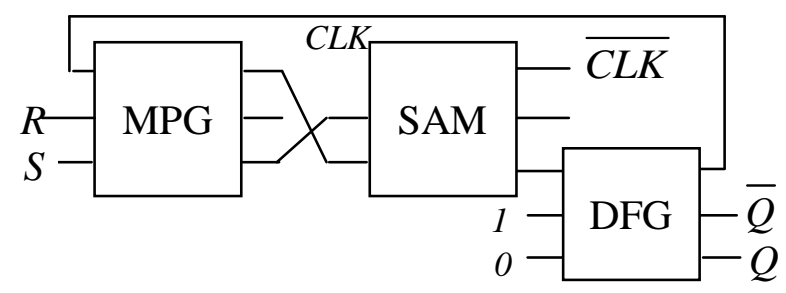

Fig. 11. Proposed design of gated SR flip-flop with $Q$ and $\bar{Q}$ outputs

Proposed gated SR flip-flop with $Q$ and $\bar{Q}$ outputs has quantum cost 10 , delay 10 and has 2 garbage bits. The proposed design of gated SR flip-flop achieves improvement ratios of $41 \%, 41 \%$ and $33 \%$ in terms of quantum cost, delay and garbage outputs compared to the design presented in Thapliyal et al.2010 [15]. The comparisons of our gated SR flip-flop (with $Q$ and $\bar{Q}$ outputs) design with existing designs in literature are summarized in Table III.

TABLE III. COMPARISONS OF DIFFERENT TYPES OF GATED SR FLIPFLOPS WITH Q AND $\bar{Q}$ OUTPUTS

\begin{tabular}{|l|c|c|c|}
\hline \multirow{2}{*}{ Gated SR flip-flop design } & \multicolumn{3}{|c|}{ Cost Comparison } \\
\cline { 2 - 4 } & Quantum Cost & Delay & $\begin{array}{c}\text { Garbage } \\
\text { Outputs }\end{array}$ \\
\hline Proposed & 11 & 11 & 2 \\
\hline Existing[15] & 17 & 17 & 3 \\
\hline Improvement in (\%) w.r.t. [15] & 41 & 41 & 33 \\
\hline
\end{tabular}

Our proposed Master Slave SR flip-flop with only $Q$ output is shown in Fig. 12.

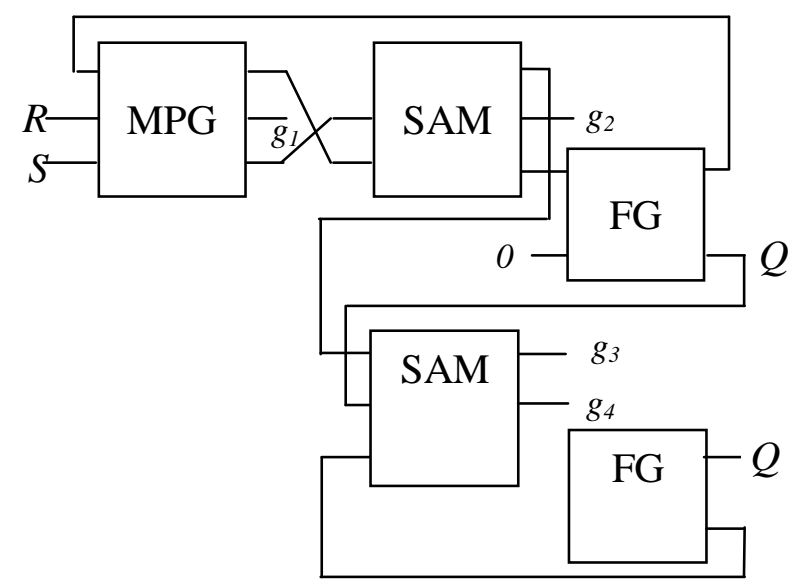

Fig. 12. Proposed Design of Master Slave SR flip-flop with only $Q$ output

The proposed master-slave SR flip-flop with only $Q$ output has quantum cost 14 , delay 14 and has 4 garbage bits. The proposed design of master slave SR flip-flop achieves improvement ratios of $36 \%$ and $36 \%$ in terms of quantum cost and delay compared to the design presented in Thapliyal et al. 2010[15]. The comparisons of our Master Slave SR flip-flop design with existing designs in literature are summarized in Table IV.

TABLE IV. COMPARISONS OF DIFFERENT TYPES OF MASTER SLAVE SR FLIP-FLOPS WITH ONLY $Q$ OUTPUT

\begin{tabular}{|l|c|c|c|}
\hline \multirow{2}{*}{$\begin{array}{c}\text { Master slave SR flip-flop } \\
\text { design }\end{array}$} & \multicolumn{3}{|c|}{ Cost Comparison } \\
\cline { 2 - 4 } & $\begin{array}{c}\text { Quantum } \\
\text { Cost }\end{array}$ & Delay & $\begin{array}{c}\text { Garbage } \\
\text { Outputs }\end{array}$ \\
\hline Proposed & 15 & 15 & 4 \\
\hline Existing[15] & 22 & 22 & 4 \\
\hline Improvement in (\%) w.r.t. [15] & 36 & 36 & 0 \\
\hline
\end{tabular}

\section{B. The JK Flip-Flop}

The characteristic equation of a JK flip-flop is $Q=J \bar{Q}+Q \bar{K}$ . The JK flip-flop is realized by one SAM gate. It can be 
mapped with the SAM gate by giving $Q, J$ and $K$ to $1^{\text {st }}, 2^{\text {nd }}$ and $3^{\text {rd }}$ inputs to the SAM gate. The proposed JK flip-flop with $Q$ and $\bar{Q}$ outputs is shown in Fig. 13 .

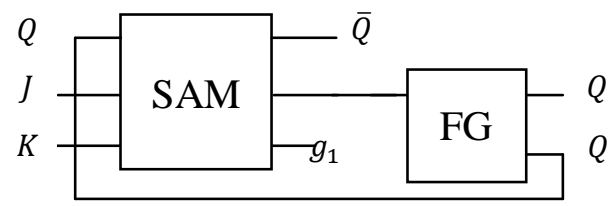

Fig. 13. Proposed design of JK flip-flop with $Q$ and $\bar{Q}$ outputs

The proposed JK flip-flop with $Q$ and $\bar{Q}$ outputs has quantum cost 5 , delay 5 and has the bare minimum of 1 garbage bit. The proposed design of JK flip-flop achieves improvement ratios of $62 \%, 62 \%$ and $67 \%$ in terms of quantum cost, delay and garbage outputs compared to the design presented in Thapliyal et al. 2010[15]. The improvement ratios compared to the design presented in Lafifa Jamal et al. $2012[16]$ are $58 \%, 58 \%$ and $67 \%$ in terms quantum cost, delay and garbage outputs. The comparisons of our JK flip-flop (with $\mathrm{Q}$ and $\bar{Q}$ outputs) design with existing designs in literature are summarized in Table V.

TABLE V. COMPARISONS OF DIFFERENT TYPES OF JK FLIP-FLOPS WITH Q AND $\bar{Q}$ OUTPUTS

\begin{tabular}{|l|c|c|c|}
\hline \multirow{2}{*}{ JK flip-flop design } & \multicolumn{3}{|c|}{ Cost Comparisons } \\
\cline { 2 - 4 } & $\begin{array}{c}\text { Quantum } \\
\text { Cost }\end{array}$ & Delay & $\begin{array}{c}\text { Garbage } \\
\text { Outputs }\end{array}$ \\
\hline Proposed & 5 & 5 & 1 \\
\hline Existing[15] & 13 & 13 & 3 \\
\hline Existing[16] & 12 & 12 & 3 \\
\hline Improvement in (\%) w.r.t. [15] & 62 & 62 & 67 \\
\hline Improvement in (\%) w.r.t. [16] & 58 & 58 & 67 \\
\hline
\end{tabular}

The characteristic equation of gated JK flip-flop is $Q=\overline{C L K} Q+C L K(J \bar{Q}+Q \bar{K})$. The gated JK flip-flop with $Q$ and $\bar{Q}$ outputs is realized by two SAM gates and one DFG. The proposed gated JK flip-flop is shown in Fig.14.

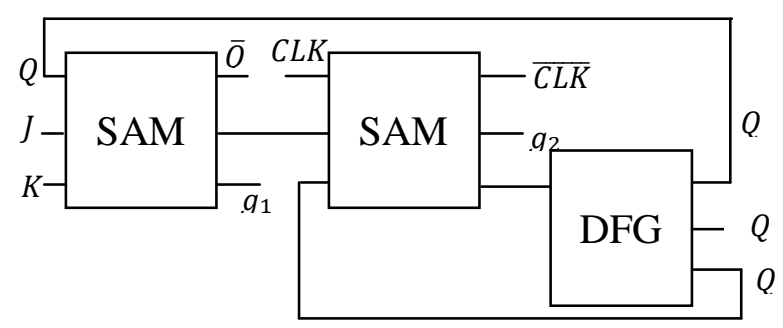

Fig. 14. Proposed Design of gated JK flip-flop with $Q$ and $\bar{Q}$ outputs.

The proposed gated JK flip-flop with $Q$ and $\bar{Q}$ outputs has quantum cost 10 , delay 10 and has 2 garbage bits. The proposed design of gated JK flip-flop achieves improvement ratios of $37 \%, 37 \%$ and $33 \%$ in terms of quantum cost, delay and garbage outputs compared to the design presented in Thapliyal and Vinod 2007[17]. The improvement ratios compared to the design presented in Thapliyal et al. 2010[15] are $23 \%, 23 \%$ and $33 \%$ in terms quantum cost, delay and garbage outputs. The comparisons of our gated JK flip-flop (with $Q$ and $\bar{Q}$ outputs) design with existing designs in literature are summarized in Table VI.

TABLE VI. COMPARISONS OF DIFFERENT TYPES OF GATED JK FLIP-FLOPS WITH $Q$ AND $\bar{Q}$ OUTPUTS

\begin{tabular}{|l|c|c|c|}
\hline \multirow{2}{*}{ Gated JK flip-flop design } & \multicolumn{3}{|c|}{ Cost Comparisons } \\
\cline { 2 - 4 } & Quantum Cost & Delay & $\begin{array}{c}\text { Garbage } \\
\text { Outputs }\end{array}$ \\
\hline Proposed & 10 & 10 & 2 \\
\hline Existing[17] & 16 & 16 & 3 \\
\hline Existing[15] & 13 & 13 & 3 \\
\hline Improvement in (\%) w.r.t. [17] & 37 & 37 & 33 \\
\hline Improvement in (\%) w.r.t. [15] & 23 & 23 & 33 \\
\hline
\end{tabular}

Our proposed Master Slave JK flip-flop with $Q$ and $\bar{Q}$ outputs is shown in Fig. 15.

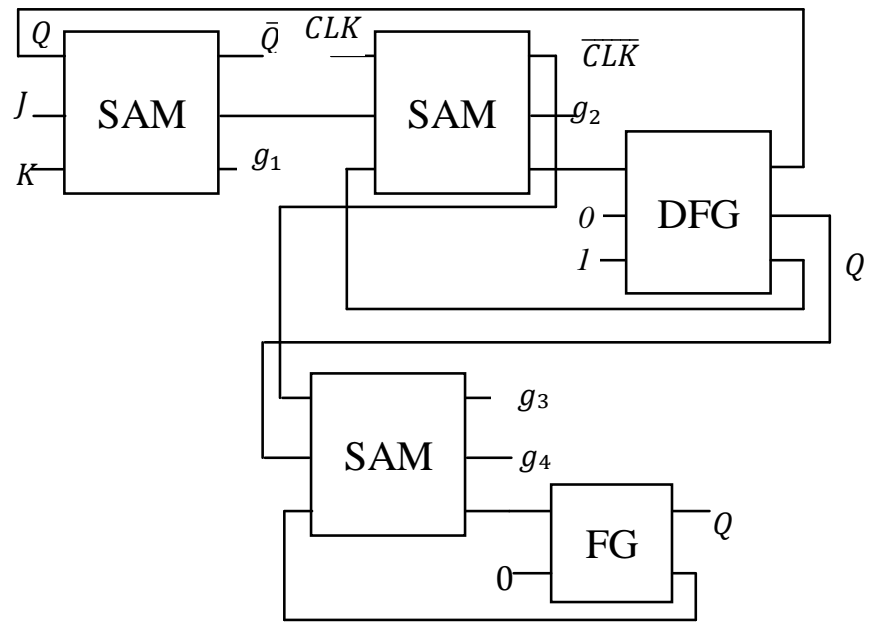

Fig. 15. Proposed Master Slave JK flip-flop with $Q$ and $\bar{Q}$ outputs

The proposed master-slave JK flip-flop with $Q$ and $\bar{Q}$ outputs has quantum cost 15 , delay 15 and has 4 garbage bits. The proposed design of master slave JK flip-flop achieves improvement ratios of $37 \%$ and $37 \%$ in terms of quantum cost and delay compared to the design presented in Thapliyal and Vonod 2007[17]. The improvement ratios compared to the design presented in Thapliyal et al. 2010[15] are 21\% and 21\% in terms quantum cost and delay. The comparisons of our master slave JK flip-flop (with $Q$ and $\bar{Q}$ outputs) design with existing designs in literature are summarized in Table VII.

TABLE VII. COMPARISONS OF DIFFERENT TYPES OF MASTER SLAVE JK FLIP-FLOPS WITH $Q$ AND $\bar{Q}$ OUTPUTS

\begin{tabular}{|l|c|c|c|}
\hline \multirow{2}{*}{$\begin{array}{c}\text { Master slave JK flip-flop } \\
\text { design }\end{array}$} & \multicolumn{3}{c|}{ Cost Comparisons } \\
\cline { 2 - 4 } & $\begin{array}{c}\text { Quantum } \\
\text { Cost }\end{array}$ & Delay & $\begin{array}{c}\text { Garbage } \\
\text { Outputs }\end{array}$ \\
\hline Proposed & 15 & 15 & 4 \\
\hline Existing[17] & 24 & 23 & 5 \\
\hline Existing[15] & 19 & 19 & 4 \\
\hline Improvement in (\%) w.r.t. [17] & 37 & 37 & 20 \\
\hline Improvement in (\%) w.r.t. [15] & 21 & 21 & 0 \\
\hline
\end{tabular}




\section{The D Flip-Flop}

The characteristic equation of gated $\mathrm{D}$ flip-flop is $Q=\overline{C L K} \cdot Q+C L K . D$. The D flip-flop can be realized by one SAM gate and one DFG. It can be mapped with SAM gate by giving CLK, D and Q respectively in $1^{\text {st }}, 2^{\text {nd }}$ and $3^{\text {rd }}$ inputs of SAM gate. The Fig. 16 shows our proposed gated D flip-flop with with $Q$ and $\bar{Q}$ outputs.

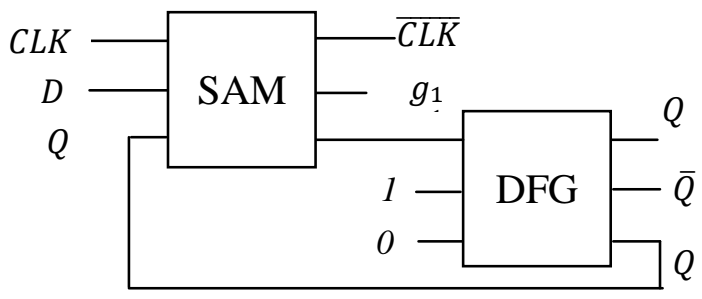

Fig. 16. Proposed design gated D flip-flop with $Q$ and $\bar{Q}$ outputs.

The proposed gated D flip-flop with $Q$ and $\bar{Q}$ outputs has quantum cost 6 , delay 6 and has the bare minimum of 1 garbage bit. The proposed design of gated D flip-flop achieves improvement ratios of $14 \%, 14 \%$ and $50 \%$ in terms of quantum cost, delay and garbage outputs compared to the design presented in Thapliyal et al. 2010[15] and Lafifa Jamal et al. 2012[16]. The comparisons of our gated D flip-flop (with $Q$ and $\bar{Q}$ outputs) design with existing designs in literature are summarized in Table VIII.

TABLE VIII. COMPARISONS OF DIFFERENT TYPES OF GATED D FLIP-FLOPS WITH Q AND $\bar{Q}$ OUTPUTS

\begin{tabular}{|l|c|c|c|}
\hline \multirow{2}{*}{\multicolumn{1}{|c|}{ D flip-flop design }} & \multicolumn{3}{c|}{ Cost Comparisons } \\
\cline { 2 - 4 } & Quantum Cost & Delay & $\begin{array}{c}\text { Garbage } \\
\text { Outputs }\end{array}$ \\
\hline Proposed & 6 & 6 & 1 \\
\hline Existing[15] & 7 & 7 & 2 \\
\hline Existing[16] & 7 & 7 & 2 \\
\hline Improvement in (\%) w.r.t. [15] & 14 & 14 & 50 \\
\hline Improvement in (\%) w.r.t. [16] & 14 & 14 & 50 \\
\hline
\end{tabular}

Our proposed Master Slave D flip-flop with $Q$ and $\bar{Q}$ outputs is shown in Fig. 17.

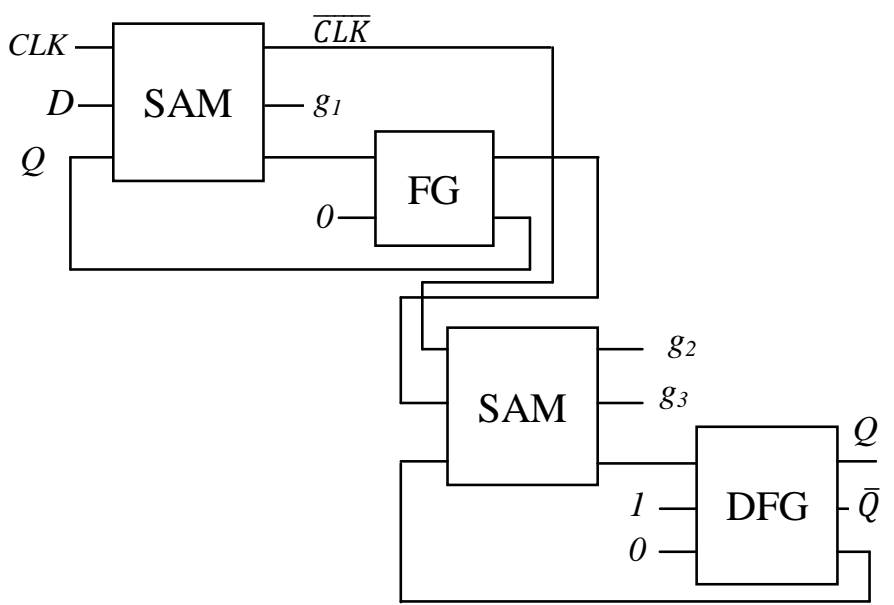

Fig. 17. Proposed design Master Slave D flip-flop with $Q$ and $\bar{Q}$ outputs.
The proposed master-slave D flip-flop with $Q$ and $\bar{Q}$ outputs has quantum cost 11 , delay 11 and has 3 garbage bits. The proposed design of master slave D flip-flop achieves improvement ratios of $21 \%$ and $21 \%$ in terms of quantum cost and delay compared to the design presented in Chuang et al. 2008[18]. The improvement ratios compared to the design presented in Thapliyal et al. 2010[15] is $21 \%$ and $21 \%$ in terms quantum cost and delay. The comparisons of our master slave D flip-flop (with $Q$ and $\bar{Q}$ outputs) design with existing designs in literature are summarized in Table IX.

TABLE IX. COMPARISONS OF DIFFERENT TYPES OF MASTER SLAVE D FLIP-FLOPS WITH $Q$ AND $\bar{Q}$ OUTPUTS

\begin{tabular}{|l|c|c|c|}
\hline \multicolumn{1}{|c|}{$\begin{array}{c}\text { Master Slave D flip-flop } \\
\text { design }\end{array}$} & \multicolumn{3}{|c|}{ Cost Comparisons } \\
\cline { 2 - 4 } & $\begin{array}{c}\text { Quantum } \\
\text { Cost }\end{array}$ & Delay & $\begin{array}{c}\text { Garbage } \\
\text { Outputs }\end{array}$ \\
\hline Proposed & 11 & 11 & 3 \\
\hline Existing[18] & 14 & 14 & 3 \\
\hline Existing[15] & 13 & 13 & 3 \\
\hline Improvement in (\%) w.r.t. [17] & 21 & 21 & 0 \\
\hline Improvement in (\%) w.r.t. [15] & 15 & 15 & 0 \\
\hline
\end{tabular}

\section{CONCLUSION}

Reversible latches are going to be the main memory block for the forthcoming quantum devices. In this paper we proposed optimized reversible D latch and JK latches with the help of proposed SAM gates. Appropriate algorithms and theorems are presented to clarify the proposed design and to establish its efficiency. We compared our design with existing ones in literature which claims our success in terms of number of gates, number of garbage outputs and delay. This optimization can contribute significantly in reversible logic community.

\section{ACKNOWLEDGMENT}

The authors would like to thank the anonymous referees for their supports and constructive feedback, which helped significantly to improve technical quality of this paper.

\section{REFERENCES}

[1] Rolf Landauer, "Irreversibility and Heat Generation in the Computing Process", IBM Journal of Research and Development, vol. 5, pp. 183191, 1961.

[2] Charles H.Bennett, "Logical Reversibility of computation", IBM Journal of Research and Development, vol. 17, no. 6, pp. 525-532, 1973.

[3] Perkowski, M., A.Al-Rabadi, P. Kerntopf, A. Buller, M. ChrzanowskaJeske, A. Mishchenko, M. Azad Khan, A. Coppola, S. Yanushkevich, V. Shmerko and L. Jozwiak, "A general decomposition for reversible logic”, Proc. RM’2001, Starkville, pp: 119-138, 2001

[4] J.E Rice, "A New Look at Reversible Memory Elements", Proceedings International Symposium on Circuits and Systems(ISCAS) 2006, Kos, Greece, May 21-24 ,2006, pp. 243-246.

[5] Dmitri Maslov and D. Michael Miller, "Comparison of the cost metrics for reversible and quantum logic synthesis", http://arxiv.org/abs/quant$\mathrm{ph} / 0511008,2006$

[6] Md. Selim Al Mamun and Syed Monowar Hossain. "Design of Reversible Random Access Memory." International Journal of Computer Applications 56.15 (2012): 18-23.

[7] Richard P.Feynman, "Quantum mechanical computers," Foundations of Physics, vol. 16, no. 6, pp. 507-531, 1986.

[8] Mohammadi,M. and Mshghi,M, On figures ofmerit in reversible and quantumlogic designs, Quantum Inform. Process. 8, 4, 297-318, 2009. 
[9] D. Michael Miller, Dmitri Maslov, GerhardW. Dueck, A Transformation Based Algorithm for Reversible Logic Synthesis, Annual ACM IEEE Design Automation Conference,Proceedings of the 40th annual Design Automation Conference, Anaheim, CA, USA Pages: 318 - 323.

[10] Perkowski, M., "A hierarchical approach to computer-aided design of quantum circuits", 6th International Symposium on Representations and Methodology of Future Computing Technology, 201-209, 2003.

[11] Tommaso Toffoli, "Reversible Computing," Automata, Languages and Programming, 7th Colloquium of Lecture Notes in Computer Science, vol. 85 , pp. 632-644, 1980.

[12] Edward Fredkin and Tommaso Toffoli, "Conservative Logic," International Journal of Theoretical Physics, vol. 21, pp. 219-253, 1982.

[13] A. Peres, "Reversible Logic and Quantum Computers," Physical Review A, vol. 32, pp. 3266-3276, 1985.

[14] J. E. Rice, An introduction to reversible latches. The Computer journal,Vol. 51, No.6, 700-709. 2008.
[15] Himanshu Thapliyal and Nagarajan Ranganathan, Design of Reversible Sequential Circuits Optimizing Quantum Cost, Delay, and Garbage Outputs, ACMJournal onEmerging Technologies inComputer Systems, Vol. 6,No. 4,Article 14, Pub. date:December 2010.

[16] Lafifa Jamal, Farah Sharmin, Md. Abdul Mottalib and Hafiz Md. Hasan Babu, Design and Minimization of Reversible Circuits for a Data Acquisition and Storage System, International Journal of Engineering and Technology Volume 2 No. 1, January, 2012

[17] H. Thapliyal and A. P. Vinod, "Design of reversible sequential elements with feasibility of transistor implementation" In Proc. the 2007 IEEE Intl. Symp. On Cir.and Sys., pages 625-628, New Orleans, USA, May 2007.

[18] M.-L. Chuang and C.-Y. Wang, "Synthesis of reversible sequential elements," ACM journal of Engineering Technologies in Computing Systems (JETC). Vol. 3, No.4, 1-19, 2008. 